\author{
Dr. Jürgen Bachmann \\ Schmerzmedizin - Orthopädie - Translationale Medizin \\ Sünsbruch 16, 45527 Hattingen, Deutschland \\ dza@dr-bachmann-hattingen.de
}

\title{
Alles hat eine andere Seite!
}

Liebe LeserInnen und KollegInnen,

einleitend möchte ich einmal die letzte Seite, die Rubrik „Die andere Seite“ an den Anfang stellen. Wie hat Ihnen die Neuerung gefallen, in unserer Zeitschrift die Patientenperspektive zu Wort kommen zu lassen?

Diese Perspektive spielt seit einigen Jahren eine Rolle in der Qualitätssicherung (z. B. für die sektorenübergreifende Qualitätssicherung der Versorgung bei Schlaganfall und Transitorische ischämische Attacke (TIA) [1]). Gerade vor dem Hintergrund der Diskussion um fragwürdige Interpretationen der Ergebnisse der Modellvorhaben in der Akupunktur ist die wissenschaftliche Untersuchung des Phänomens „Placebo“ früh angeregt und ein Paradigmenwechsel angestoßen worden, der den Effekten der Patientenerwartungen eine zentrale Rolle zuerkennt. Diese Effekte können beeinflusst werden! Sie sind auf neuropsychologische Phänomene zurückzuführen und tragen zum Behandlungsergebnis bei. Im Weiteren bestimmen gerade bei chronischen und progredienten Erkrankungen die Vorstellungen der Patienten und ihre Verarbeitungsmechanismen die Entwicklung vom Krankheitsbild zur Gesundheit.

Ich bin mir sicher, dass sich in der Klientel der Leserschaft Personen finden lassen, die lesenswerte, interessante oder erfolgreiche Beispiele für eine gelungene Krankheitsverarbeitung auf dem Boden der individuellen Ressourcen liefern können. Bringen Sie sich ein!

Was bringen wir Ihnen in dieser schlanken Ausgabe?

Die Übersichtsarbeit „Erfahrung und Wissenschaft in Tradition und Moderne“ unter Federführung von K. Helbig widmet sich in dem zweiten von 3 Teilen den Ergebnissen eines strukturierten Diskurses zwischen Vertretern verschiedener Fachdisziplinen der „Entwicklung des Lebens zum subjektiven Selbst“.

Die aktuellen Zahlen der Akupunkturpublikationen (Randomized Controlled Trial (RCTs), systematische Reviews und Metaanalysen) steigt - keine Überraschung, aber ein immer schwerer wiegendes Argument für die Diskussion mit den Leugnern. In der Rubrik Wissenschaft für die Kitteltasche zeigen uns K. Hua und J. Dietzel aus dem Projektbereich komplementäre und inte- grative Medizin der Charité den Forschungsstand zur peripheren Fazialisparese. Im Journal Club widmet sich S. Eisenhardt der Ohrakupunktur bei Kindern mit AufmerksamkeitsdefizitHyperaktivitätsstörung (ADHS).

Der Praxisteil widmet sich unter Federführung von D. Irnich schwerpunktmäßig einem methodischen Thema: Die Elektroakupunktur ist gut belegt und bietet ein breites Anwendungsspektrum.

C. Tauber-Bachmann erläutert in ihrer Kolumne Erkenntnisse zur Prävention aus dem vergangenen Lockdown, im Hinblick auf die kommende Wintersaison Grundlage für proaktive Überlegungen, und C. Chiu teilt anhand einer Darstellung über 4 Fälle Effektives zur Infektbehandlung mit.

Die Auswahl kommentierter News hat A. Wiebrecht in bewährter Weise für Sie zusammengestellt. G. Linck ist wie kaum eine andere berufen, einen Nachruf auf Heribert Schmidt zu formulieren.

Viel Erfolg wünscht Ihnen

Jürgen Bachmann

Interessenkonflikt. J. Bachmann gibt an, dass kein Interessenkonflikt besteht. 